\title{
A unified Green's function analysis of complicated DFB lasers
}

Jim D. Freeze

byu@freeze.org

Michael A. Jensen

jensen@byu.edu

Richard H. Selfridge

selfridge@ee.byu.edu

Follow this and additional works at: https://scholarsarchive.byu.edu/facpub

Part of the Electrical and Computer Engineering Commons

\section{Original Publication Citation}

Freeze, J. D., M. A. Jensen, and R. H. Selfridge. "A Unified Green's Function Analysis of Complicated DFB Lasers." Quantum Electronics, IEEE Journal of 33.8 presented for evaluation of distributed feedback (DFB) laser cavities with arbitrary material profiles. The method first constructs the Green's function of an inhomogeneous domain and subsequently uses Green's theorem to determine the laser o(TRUNCATED) (1997): 1253-9

\section{BYU ScholarsArchive Citation}

Freeze, Jim D.; Jensen, Michael A.; and Selfridge, Richard H., "A unified Green's function analysis of complicated DFB lasers" (1997). Faculty Publications. 664.

https://scholarsarchive.byu.edu/facpub/664

This Peer-Reviewed Article is brought to you for free and open access by BYU ScholarsArchive. It has been accepted for inclusion in Faculty Publications by an authorized administrator of BYU ScholarsArchive. For more information, please contact ellen_amatangelo@byu.edu. 


\title{
A Unified Green's Function Analysis of Complicated DFB Lasers
}

\author{
Jim D. Freeze, Member, IEEE, Michael A. Jensen, Member, IEEE, and Richard H. Selfridge, Member, IEEE
}

\begin{abstract}
An efficient full-wave analysis technique for onedimensional optical domains, known as the Recursive Green's Function Method (RGFM), is presented for evaluation of distributed feedback (DFB) laser cavities with arbitrary material profiles. The method first constructs the Green's function of an inhomogeneous domain and subsequently uses Green's theorem to determine the laser optical field, lasing wavelength, and threshold gain. The technique is applied to investigate the performance of three DFB laser structures: a chirped-grating configuration, a modulated stripe width design, and a reduced duty cycle complexcoupled device. These structures are evaluated in terms of their single-mode lasing behavior and the uniformity of the optical field within the cavity.
\end{abstract}

Index Terms-Distributed feedback lasers, Green's functions, nonhomogenous media, periodic structures, semiconductor lasers.

\section{INTRODUCTION}

D ISTRIBUTED feedback (DFB) plays an increasingly important role in both analog and digital communications environments. This is particularly true in semiconductor lasers and in fiber lasers and amplifiers where DFB preferentially enhances gain for a single longitudinal mode. The resulting single-mode behavior alleviates problems associated with multimode dispersion and allows large modulation bandwidths. However, several challenges exist in designing stable singlemode oscillators, such as mode degeneracy in index-coupled structures and nonlinearities due to spatial hole burning (SHB). In order to examine and compare the performance of different laser geometries, it is necessary to have analysis tools which accurately predict the behavior of arbitrary material profiles in the cavity.

Typical analyses for evaluating DFB laser behavior make use of a coupled-mode analysis in which approximate differential equations are formulated and solved for the optical fields [1]. Such an approach has proven highly effective for purely periodic structures and has been used with some success for nearly periodic configurations such as chirped or tapered gratings. However, this technique does of necessity rely on simplifying approximations, and therefore can compromise computational accuracy, particularly for nonperiodic structures [2]. Additionally, the coupled-mode analysis requires reformulation of the governing differential equations and their solutions each time a different material profile is introduced.

Manuscript received April 16, 1996; revised January 31, 1997.

The authors are with the Department of Electrical and Computer Engineering, Brigham Young University, Provo, UT 84602 USA.

Publisher Item Identifier S 0018-9197(97)05450-X.
In this paper, we present a simple, full-wave onedimensional (1-D) analysis technique which allows efficient simulation of laser cavities with virtually any material distribution. This approach, known as the Recursive Green's Function Method (RGFM), is based upon a previously reported technique [2]-[4]. However, we extend the method to allow determination of the fields within the optical device as well as provide a more rigorous presentation of the theory. Unlike typical full-wave analysis techniques, the RGFM provides excellent computational and storage efficiency. Additionally, due to its unique recursive formulation, it is highly suitable for periodic or nearly periodic structures such as DFB laser devices. While the method represents a general analysis tool for arbitrary inhomogeneous optical domains, we present it as a unified flexible approach for fast and accurate analysis of different DFB laser configurations.

It is noteworthy that the RGFM formulated here is very similar in concept to the Transfer Matrix Method (TMM) [5], [6] which has received considerable attention as a viable optical device analysis technique. Therefore, the RGFM represents an alternative scheme with comparable computational efficiencies, flexibilities, and capabilities. The major difference between the two approaches is that the TMM provides the laser response using chain or transfer matrices while the RGFM uses the Green's function describing the device. The key advantage of this latter approach is that, due to the fact that Green's functions can be defined and utilized in one, two, or three dimensions, the simple concepts presented here for a 1-D analysis can be extended to algorithms in higher dimensions using straightforward generalizations [7]. This extension and its application to optical domains will be the subject of a future correspondence.

The utility and flexibility of the RGFM are demonstrated by its application to several practical and recently proposed DFB laser structures which are difficult to analyze with simple approximate formulations. First, the RGFM is used to investigate two nonperiodic devices: a chirped-grating (CG) DFB geometry [8], [9] and a modulated stripe width (MSW) configuration [10], [11]. The performance of these structures is compared to that of a quarter-wave-shifted (QWS) laser [12], [13] in terms of single-mode gain margin - the threshold gain difference between the main lasing mode and the next most significant side mode - and the SHB. Additionally, a complexcoupled DFB configuration is investigated in which the duty cycle of the grating is altered. The results confirm a recently published finding [14] that such a scheme can noticeably enhance the gain margin of complex-coupled devices. 


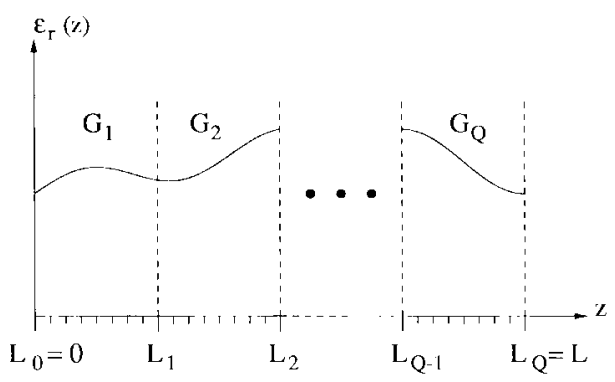

Fig. 1. 1-D domain with inhomogeneous relative permittivity divided into $Q$ unit sections.

\section{THEORY}

\section{A. RGFM Derivation}

The RGFM is a fast full-wave solution method for computing the fields in a 1-D domain with arbitrary material inhomogeneity. Consider a domain $\Omega$ characterized by the complex relative permittivity $\epsilon_{r}(z)$. The field intensity $E(z)$ inside this domain obeys the scalar Helmholtz equation given as

$$
\left[\nabla^{2}+k_{\sigma}^{2} \epsilon_{r}(z)\right] E(z)=0, \quad z \in \Omega
$$

where $k_{\sigma}$ is the free-space wavenumber. To solve (1) for $E(z)$, consider that the domain $\Omega$ is divided into $Q$ subdomains, as implied in Fig. 1. For simplicity, we will limit the discussion to two subdomains and generalize to more sections later. We assume that the Green's functions $G_{1}$ and $G_{2}$ for (1) are known on the subdomains $\Omega_{1}$ and $\Omega_{2}$, where $\Omega=\Omega_{1}\left\llcorner\Omega_{2}\right.$ and the interface occurs at the point $z_{B}=\Omega_{1} \Gamma \Omega_{2}$. By definition, these Green's functions satisfy the equation

$$
\left[\nabla^{2}+k_{o}^{2} \epsilon_{r}(z)\right] G_{i}\left(z, z^{\prime}\right)=-\delta\left(z-z^{\prime}\right), \quad z, z^{\prime} \in \Omega_{i}
$$

for $i=1,2$, where $\delta(\cdot)$ represents the Dirac delta function. At the endpoints of the domain $\Omega_{i}$, the function $G_{i}$ can in general satisfy any boundary condition, although in this work homogeneous Neumann conditions are chosen to simplify the RGFM implementation. Methods for obtaining these Green's functions are discussed in Section II-B.

The objective of the RGFM is to combine $G_{1}$ and $G_{2}$ into a composite Green's function $G^{c}$ valid for the domain $\Omega$. Let $G_{i j}^{c}$ be defined such that

$$
G_{i j}^{c}\left(z_{i}, z_{j}\right)=G^{c}\left(z_{i}, z_{j}\right), \quad z_{k} \in \Omega_{k} .
$$

With this representation, we first observe that $G_{i i}^{c}$ satisfies the same differential equation as $G_{i}, i=1,2$, but must satisfy different boundary conditions at $z_{B}$ (since the homogeneous Neumann condition at $z_{B}$ is clearly invalid for $G^{c}$ ). This fact allows us to form $G_{i i}^{c}$ from $G_{i}$ by adding to it a homogeneous solution of (2) which allows proper manipulation of the boundary conditions. Similarly, $G_{i j}^{c}, i \neq j$, must be a homogeneous solution of (2) in its first argument. As discussed in the Appendix, these homogeneous solutions can be constructed from the $G_{i}$, resulting in the forms

$$
\begin{aligned}
& G_{11}^{c}\left(z, z^{\prime}\right)=G_{1}\left(z, z^{\prime}\right)+G_{1}\left(z, z_{B}\right) A_{11}\left(z^{\prime}\right) \\
& G_{12}^{c}\left(z, z^{\prime}\right)=G_{1}\left(z, z_{B}\right) A_{12}\left(z^{\prime}\right)
\end{aligned}
$$

$$
\begin{aligned}
& G_{21}^{c}\left(z, z^{\prime}\right)=G_{2}\left(z, z_{B}\right) A_{21}\left(z^{\prime}\right) \\
& G_{22}^{c}\left(z, z^{\prime}\right)=G_{2}\left(z, z^{\prime}\right)+G_{2}\left(z, z_{B}\right) A_{22}\left(z^{\prime}\right)
\end{aligned}
$$

where $A_{i j}$ represents an unknown function.

To complete the solution for $G^{c}$, we must apply appropriate continuity conditions for $G_{i j}^{c}$ and solve for the unknown $A_{i j}$. These conditions assume the form [15]

$$
\begin{aligned}
G_{11}^{c}\left(z_{B}, z_{1}\right) & =G_{21}^{c}\left(z_{B}, z_{1}\right) \\
\frac{\partial}{\partial z} G_{11}^{c}\left(z_{B}, z_{1}\right) & =\frac{\partial}{\partial z} G_{21}^{c}\left(z_{B}, z_{1}\right) \\
G_{22}^{c}\left(z_{B}, z_{2}\right) & =G_{12}^{c}\left(z_{B}, z_{2}\right) \\
\frac{\partial}{\partial z} G_{22}^{c}\left(z_{B}, z_{2}\right) & =\frac{\partial}{\partial z} G_{12}^{c}\left(z_{B}, z_{2}\right)
\end{aligned}
$$

where $z_{i} \in \Omega_{i}$. When using these boundary conditions on (4)-(7), however, care must be exercised when evaluating the required derivatives. For example, consider (9) which results in the expression

$$
\begin{aligned}
& \frac{\partial}{\partial z} G_{1}\left(z_{B}, z_{1}\right)+\lim _{\epsilon \rightarrow 0} \frac{\partial}{\partial z} G_{1}\left(z_{B}-\epsilon, z_{B}\right) A_{11}\left(z_{1}\right) \\
& =\lim _{\epsilon \rightarrow 0} \frac{\partial}{\partial z} G_{2}\left(z_{B}+\epsilon, z_{B}\right) A_{21}\left(z_{1}\right) .
\end{aligned}
$$

Application of the homogeneous Neumann boundary condition causes the first term of (12) to vanish. The other derivatives must be determined using the jump condition for (2), namely [15]

$$
\lim _{\epsilon \rightarrow 0}\left\{\frac{\partial}{\partial z} G_{i}\left(z^{\prime}+\epsilon, z^{\prime}\right)-\frac{\partial}{\partial z} G_{i}\left(z^{\prime}-\epsilon, z^{\prime}\right)\right\}=-1 .
$$

If we let $z^{\prime} \rightarrow z_{B}$ and again use the homogeneous Neumann boundary condition, we obtain

$$
\begin{aligned}
& \left.\lim _{\epsilon \rightarrow 0} \frac{\partial}{\partial z} G_{1}\left(z, z_{B}\right)\right|_{z=z_{B}-\epsilon}=1 \\
& \left.\lim _{\epsilon \rightarrow 0} \frac{\partial}{\partial z} G_{2}\left(z, z_{B}\right)\right|_{z=z_{B}+\epsilon}=-1
\end{aligned}
$$

which, when placed into (12), results in the condition $A_{11}\left(z_{1}\right)=-A_{21}\left(z_{1}\right)$. Using this result in conjunction with (8) results in the expression

$$
\begin{aligned}
A_{11}\left(z_{1}\right) & =-A_{21}\left(z_{1}\right) \\
& =-\mathbf{T} G_{1}\left(z_{B}, z_{1}\right)
\end{aligned}
$$

where

$$
\mathbf{T}=\left[G_{1}\left(z_{B}, z_{B}\right)+G_{2}\left(z_{B}, z_{B}\right)\right]^{-1} .
$$

A similar analysis reveals that

$$
\begin{aligned}
A_{22}\left(z_{2}\right) & =-A_{12}\left(z_{2}\right) \\
& =-\mathbf{T} G_{2}\left(z_{B}, z_{2}\right) .
\end{aligned}
$$

Finally, substitution of (16) and (18) into (4)-(7) results in the equations

$$
\begin{aligned}
& G_{11}^{c}\left(z, z^{\prime}\right)=G_{1}\left(z, z^{\prime}\right)-G_{1}\left(z, z_{B}\right) \mathbf{T} G_{1}\left(z_{B}, z^{\prime}\right) \\
& G_{12}^{c}\left(z, z^{\prime}\right)=G_{1}\left(z, z_{B}\right) \mathbf{T} G_{2}\left(z_{B}, z^{\prime}\right) \\
& G_{21}^{c}\left(z, z^{\prime}\right)=G_{2}\left(z, z_{B}\right) \mathbf{T} G_{1}\left(z_{B}, z^{\prime}\right) \\
& G_{22}^{c}\left(z, z^{\prime}\right)=G_{2}\left(z, z^{\prime}\right)-G_{2}\left(z, z_{B}\right) \mathbf{T} G_{2}\left(z_{B}, z^{\prime}\right) .
\end{aligned}
$$


Equations (19)-(22) are similar to those given in [2]. However, these results are more general in that they allow $z$ and $z^{\prime}$ to assume any value within the region rather than being restricted to the domain boundaries. This additional flexibility allows determination of the fields within the inhomogeneous domain and, therefore, provides information on the degree of SHB encountered in the device under investigation.

The recursive equations derived above imply a method for systematically constructing the Green's function for an arbitrary domain. Let the function $G_{i}^{c}$ represent the composite Green's function formed from Sections 1 through $i$ (see Fig. 1). The Green's function may then be constructed from the $Q$ unit sections using the algorithm

$$
\begin{aligned}
G_{i}^{c} & =G_{i-1}^{c} \oplus G_{i} \\
G_{1}^{c} & =G_{1}
\end{aligned}
$$

for $2 \leq i \leq Q$, where " $\oplus$ " denotes using (19)-(22) to combine two Green's functions. This simple procedure begins at the left and recursively computes the composite Green's function for the entire domain.

\section{B. Unit Section Green's Function}

Computation of the unit section Green's functions $G_{i}$ requires solution of (2) subject to homogeneous Neumann boundary conditions on the endpoints of the domain $\Omega_{i}$. For a constant permittivity $\epsilon_{r}(z)=\epsilon_{r i}=n_{i}^{2}$ for $z \in \Omega_{i}$, this Green's function is expressed as

$$
\begin{aligned}
& G_{i}\left(z>z^{\prime}\right)=-\frac{\cos \left[k_{i}\left(z-L_{i}\right)\right] \cos \left[k_{i}\left(z^{\prime}-L_{i-1}\right)\right]}{k_{i} \sin \left[k_{i}\left(L_{i}-L_{i-1}\right)\right]} \\
& G_{i}\left(z<z^{\prime}\right)=-\frac{\cos \left[k_{i}\left(z^{\prime}-L_{i}\right)\right] \cos \left[k_{i}\left(z-L_{i-1}\right)\right]}{k_{i} \sin \left[k_{i}\left(L_{i}-L_{i-1}\right)\right]}
\end{aligned}
$$

where $k_{i}=k_{o} n_{i}$ and $L_{i}$ is defined in Fig. 1. Given this Green's function representation, the computation of $G_{i}$ can proceed in two manners. First, if it is assumed that each section $\Omega_{i}$ is small, then the permittivity $\epsilon_{r}(z)$ within section $i$ can be assumed to be constant and (24) can be used directly. If larger unit sections are chosen, then (24) must be used in conjunction with Dyson's equation and a suitable integral equation solution technique to obtain the Green's function for the domain $\Omega_{i}$ [2]. While this latter case requires additional computational overhead, it allows more accurate representation of continuously varying permittivity profiles. However, for most cases, the simpler constant permittivity assumption is adequate and is, therefore, the technique used in the results presented here.

\section{Laser Threshold and Field Evaluation}

The preceding developments illustrate the construction of the Green's function given a material configuration. However, in the analysis of devices such as DFB lasers, it is necessary to determine the proper gain and wavelength at which lasing occurs. Within the RGFM framework, this is accomplished through proper implementation of Green's theorem. To see this, consider the fields external to the 1-D domain $\Omega$, as shown

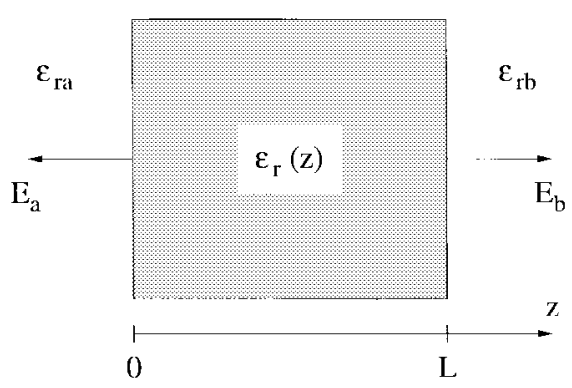

Fig. 2. A general inhomogeneous laser cavity with output fields $E_{a}$ and $E_{b}$.

in Fig. 2, which may be expressed as

$$
\begin{aligned}
& E(z \leq 0)=E_{a} e^{j k_{a} z} \\
& E(z \geq L)=E_{b} e^{-j k_{b}(z-L)}
\end{aligned}
$$

where $k_{p}=k_{o} \sqrt{\epsilon_{r p}}, p=a, b$. Given the fact that the fields must be continuous at the laser boundaries $z=0, L$, the use of Green's second identity [16] in conjunction with (1) and (2) provides the equation

$$
\begin{aligned}
E(z) & =-\left[G^{c}\left(z, z^{\prime}\right) \frac{d E\left(z^{\prime}\right)}{d z^{\prime}}-E\left(z^{\prime}\right) \frac{d G^{c}\left(z, z^{\prime}\right)}{d z^{\prime}}\right]_{E^{\prime}=0}^{L} \\
& =-j k_{b} G^{c}(z, L) E_{b}-j k_{a} G^{c}(z, 0) E_{a}
\end{aligned}
$$

where the homogeneous Neumann conditions have been applied at the domain boundaries.

The next step in the development requires evaluation of (27) at the domain boundaries $z=0, L$. Proper arrangement of the resulting equations results in the system

$$
\begin{array}{r}
{\left[1+j k_{a} G^{c}(0,0)\right] E_{a}+j k_{b} G^{c}(0, L) E_{b}=0} \\
j k_{a} G^{c}(L, 0) E_{a}+\left[1+j k_{b} G^{c}(L, L)\right] E_{b}=0
\end{array}
$$

which may be expressed as $\mathbf{A} \bar{E}=0$. This system has a nontrivial solution only if $\operatorname{det}(\mathbf{A})=0$, or

$$
\begin{aligned}
{\left[1+j k_{a} G^{c}(0,0)\right][1} & \left.+j k_{b} G^{c}(L, L)\right] \\
& +k_{a} k_{b} G^{c}(0, L) G^{c}(L, 0)=0 .
\end{aligned}
$$

Therefore, determination of laser threshold is equivalent to finding the gain and wavelength which produce a Green's function $G^{c}$ which satisfies (29). Once the gain/wavelength pair has been determined, one of the two output field values $E_{a}$ or $E_{b}$ must be specified. The remaining output field may be computed using one of the two equations represented in (28). Computation of the fields internal to the laser can then be performed using (27).

\section{EFFICIENT COMPUTER IMPLEMENTATION}

The RGFM as developed provides an efficient mechanism for analyzing the behavior of optical fields in complex domains. However, in many cases, the computational efficiency can be improved by exploiting certain geometrical and algorithmic features. The following techniques illustrate implementation details which can be used for this purpose. 


\section{A. Storage Reduction}

Examination of (27) reveals that evaluation of the fields for most laser structures requires knowledge only of the Green's functions for the source point $z^{\prime}$ at 0 and $L$. Careful examination of the expressions in (19)-(22) shows that to compute these Green's function values, the contributing Green's functions $G_{1}$ and $G_{2}$ must be known only for source points on their respective domain boundaries. This key observation allows considerable reduction of the computational and storage requirements of the RGFM. It is noteworthy that if only the fields external to the domain are desired, then the Green's functions must be computed only for the observation point $z$ at 0 and $L$ as well.

\section{B. Recursive Doubling}

Despite the simplicity and generality of the RGFM algorithm proposed in (23), implementation of the algorithm in such a fashion is not optimal. A better approach is to divide the $Q$ unit sections into nearest-neighbor pairs and combine each pair to form $Q / 2$ new Green's functions. This section-doubling procedure is then repeated recursively until the composite Green's function for the entire domain has been constructed. If the unit sections are computed using the constant-permittivity assumption as outlined in Section II-B and the observations in Section III-A are exploited, the storage and computational complexities of RGFM are given, respectively, as $O(N)$ and $O\left(N \log _{2} N\right)$, where $N$ is the total number of points in the domain. If only the fields external to the domain are required, then the Green's function must be evaluated only at the points $z, z^{\prime}=0, L$, resulting in a computational complexity of $O(N)$.

\section{Periodic Domains}

It is noteworthy that for periodic domains where the unit sections are chosen to correspond to the permittivity profile period, the Green's functions for the sections are identical. In this case, the computation can be efficiently performed by first constructing a Green's function representing one period of the geometry. With this as a fundamental building block, the technique can be used to recursively double the block by combining it with itself. In the notation of (23), we can write

$$
\begin{aligned}
G_{2 n}^{c} & =G_{n}^{c} \oplus G_{n}^{c} \\
G_{1}^{c} & =G_{1} \\
n & =2^{m}, \quad m=0,1,2, \cdots .
\end{aligned}
$$

This simple algorithm requires a computational complexity of $O(N)$ for computation of internal fields and $O\left(\log _{2} N\right)$ for external fields. For many DFB structures, this periodic doubling procedure can be used to efficiently compute the laser behavior.

\section{Study of DFB LASER DeVices}

Perhaps the single most important feature of the RGFM outlined here is its flexibility in modeling virtually any laser cavity configuration, including nonperiodic structures. To demonstrate this flexibility, we apply the method in this section to the analysis of three DFB configurations: a chirped-grating geometry, a modulated stripe width laser, and a reduced duty cycle complex-coupled device. For simplicity, it is assumed that all geometries are coated with a perfect antireflection coating on both device ends. These examples represent geometries which are difficult to model using approximate formulations such as coupled-wave theory. This analysis focuses on such issues as the single-mode gain margin and the degree of SHB encountered in the device.

Before applying the RGFM to representative practical DFB laser structures, it was necessary to ensure the computational accuracy of the method. This was tested in two manners. First, the RGFM was applied to a simple two-layer dielectric slab configuration where one of the layers was lossless while the other contained either a loss or gain term. In each case, it was found that the RGFM solution matched the results from the analytic solution to within computational precision. Second, the scheme was used to determine the threshold gain and wavelength for a simple Fabry-Perot laser cavity formed from a cleaved homogeneous gain medium. Again, the RGFM results matched those obtained using a closed-form analysis to within the precision of the root-finding algorithm used to solve (29). These simple tests provided confidence that the method should work well for other more complicated geometries.

\section{A. Chirped-Grating Index-Coupled DFB}

As a first testbed configuration, we chose a chirped-grating (CG) DFB configuration. This particular geometry has been suggested in the literature as one which removes the mode degeneracy characteristic of index-coupled DFB structures and, therefore, is much like the commonly used quarter-waveshifted (QWS) structures. However, it has been suggested that the CG topology can be designed for reduced spatial variation of the optical field intensity within the cavity and is therefore less likely to suffer from nonlinearities induced by SHB [8], [9].

For this computation, we chose a $L=310-\mu$ m-long DFB structure whose grating pitch $\Lambda$ was tapered linearly over the length of the cavity as

$$
\Lambda=\Lambda_{o}-\xi|z|, \quad-\frac{L}{2} \leq z \leq \frac{L}{2} .
$$

The refractive index grating profile is modeled as

$$
n(z)=\sqrt{\epsilon_{r}(z)}=n_{a}+\Delta n \sin \left(\frac{2 \pi}{\Lambda} z\right)+j \frac{g}{2 k_{o}}
$$

where $g$ is the material intensity gain. In this computational model, we have chosen $n_{a}=3.5977$ and $\Lambda_{o}=0.2154 \mu \mathrm{m}$ which produce a Bragg wavelength (for $\xi=0$ ) of $\lambda_{B}=$ $1.55 \mu \mathrm{m}$. We will use the common parameter

$$
\kappa=\frac{\pi \Delta n}{\lambda_{B}}
$$

to characterize the grating depth.

As a first study, consider the threshold gain difference $\Delta g_{\text {th }}$ between the mode with the lowest threshold gain and the most significant side mode. Fig. 3 plots this value versus the chirping parameter $\xi$ for three different values of $\kappa L$. 


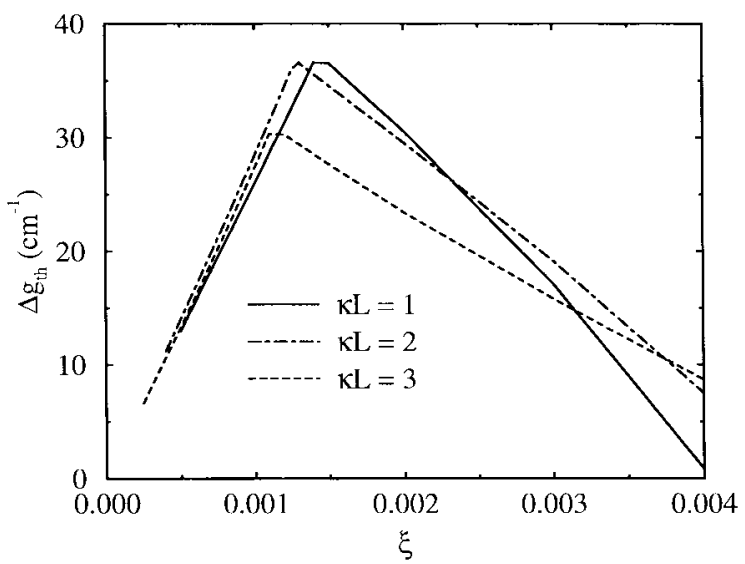

Fig. 3. Single-mode gain margin $\Delta g_{\text {th }}$ versus the chirping parameter $\xi$ for the CG DFB cavity at three different values of $\kappa L$.

TABLE I

COMPARISON OF $\Delta g_{\text {th }}$ AND CF FOR THE CG, MSW, AND QWS DFB Structures for Three Different Values of $\kappa L$

\begin{tabular}{l|cc|cc|cc}
\hline \hline & \multicolumn{2}{|c}{ CG DFB } & \multicolumn{2}{c}{ MSW DFB } & \multicolumn{2}{c}{ QWS DFB } \\
$\kappa L$ & $\Delta g_{t h}\left(\mathrm{~cm}^{-1}\right)$ & $\mathrm{CF}$ & $\Delta g_{t h}\left(\mathrm{~cm}^{-1}\right)$ & $\mathrm{CF}$ & $\Delta g_{\text {th }}\left(\mathrm{cm}^{1}\right)$ & $\mathrm{CF}$ \\
\hline 1 & 36.6 & 0.587 & 39.0 & 0.456 & 44.1 & 0.655 \\
2 & 36.6 & 0.418 & 43.1 & 0.471 & 47.0 & 0.243 \\
3 & 30.3 & 0.188 & 40.7 & 0.178 & 42.3 & 0.062 \\
\hline \hline
\end{tabular}

As can be seen, there is clearly an optimal choice of the chirping parameter for maximization of the threshold gain difference. Table I compares the values of $\Delta g_{\text {th }}$ for the CG structure with those for the QWS cavity with the same physical parameters. Also included are the results for the modulated stripe width (MSW) laser considered in Section IV-B. These results indicate that while the CG configuration provides good single-mode gain margin, for this particular configuration it does not perform as well as the simple QWS structure.

In contrast, it is interesting to consider the degree of SHB encountered within the CG DFB cavity. As a quantitative measure of the SHB, the term $\mathrm{CF}$ is used to denote the ratio of the minimum to maximum optical field intensity along the length of the cavity. To compare with similar studies which have been performed, the optical field is first processed with a moving average filter with a window length equal to the period of the field standing wave to remove the standing wave variations. The comparison of the $\mathrm{CF}$ values in Table I illustrates that for weak coupling (small $\kappa L$ ), the QWS structure suffers from less hole burning. However, as $\kappa L$ is increased, the CG geometry appears to outperform the QWS laser for these parameters. Perhaps more important is the fact that if a lower value of $\Delta g_{\mathrm{th}}$ can be tolerated, then the hole burning can be reduced even further for the CG configuration. This is illustrated in Fig. 4, which plots the CF value as a function of $\xi$ for the three values of $\kappa L$. Here, it can be seen that for higher values of $\kappa L$ choosing a lower value of $\xi$ than that which provides optimal gain margin results in an increased value of CF. Fig. 5 illustrates the field variation in the cavity at $\kappa L=2$ for three different values of $\xi$ and for the QWS structure. This plot illustrates the peaked nature of the field

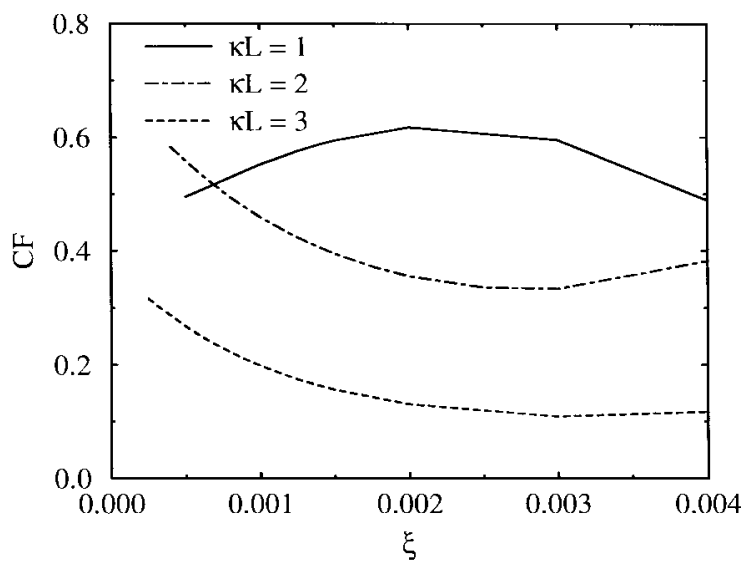

Fig. 4. CF number versus the chirping parameter $\xi$ for the CG DFB cavity at three different values of $\kappa L$.

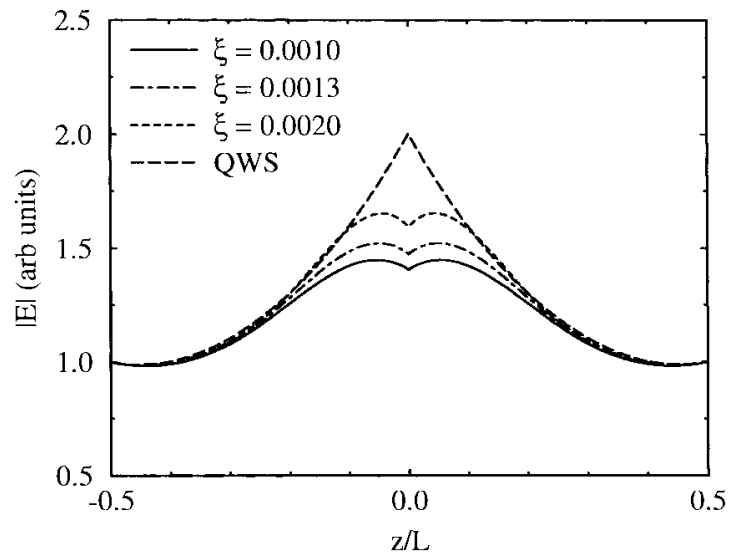

Fig. 5. Optical field strength versus position in the CG laser structure for three different values of $\xi$ compared with the field for the QWS structure. All computations use $\kappa L=2$.

in the QWS structure which results in pronounced SHB for strongly coupled configurations.

\section{B. MSW Index-Coupled DFB}

An alternative structure which can remove the mode degeneracy suffered by index-coupled DFB lasers is the MSW geometry [10], [11]. In this configuration, a short section in the middle of the cavity has a slightly higher refractive index $n_{a}$ than the remainder of the cavity. The same basic cavity parameters as given in Section IV-A were again used, with the exception that a $43-\mu \mathrm{m}$-long section in the center of the cavity was assigned a refractive index of $n_{a}^{\prime}=3.6067$. For this configuration, the gain difference $\Delta g_{\mathrm{th}}$ and the SHB parameter $\mathrm{CF}$ are listed in Table I for three values of $\kappa L$. As can be seen, the MSW laser has a slightly higher threshold gain difference $\Delta g_{\mathrm{th}}$ than the CG configuration but slightly lower value than the QWS structure. Much like the CG geometry, the MSW offers improved SHB performance for higher coupling strengths as compared with the QWS topology. The spatial field profiles for the three values of $\kappa L$ for the MSW and QWS structures are compared in Fig. 6. Once again, we see that the MSW field profile is less peaked than the QWS structure which implies a reduced effect of SHB on the laser performance. 


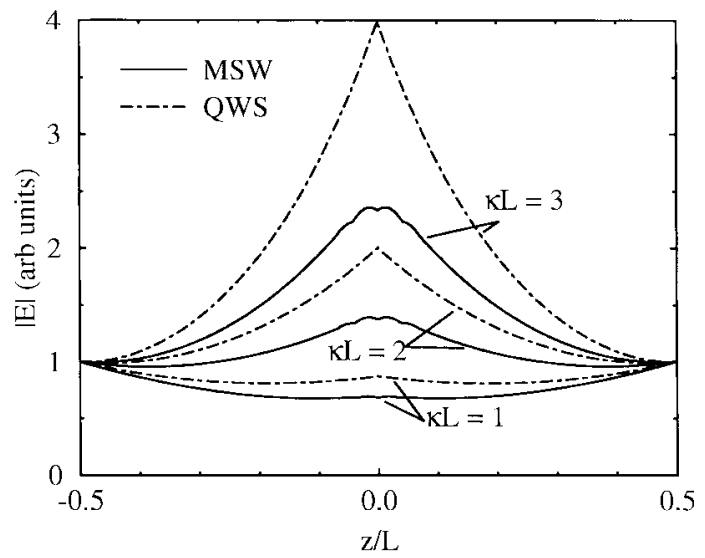

Fig. 6. Optical field strength versus position in the MSW and QWS laser structures for three different values of $\kappa L$.

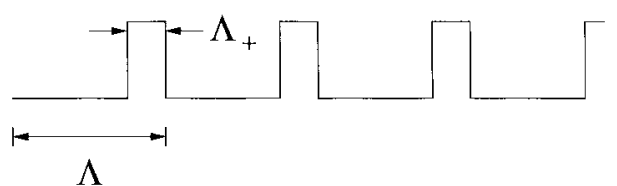

Fig. 7. Complex grating profile with high gain/high index region length $\Lambda_{+}$.

\section{Reduced Duty Cycle Complex-Coupled DFB}

As a final example, we consider a recently proposed complex-coupled structure designed for improved single-mode gain margin [14]. In this structure, we will let the index and gain modulations assume a square rather than sinusoidal wave shape for simplicity. The unique feature of this geometry is the fact that the length $\Lambda_{+}$of the high gain/high index grating region is less than half the grating period $\Lambda$, as implied in Fig. 7. We will use the model that the spatially varying field gain $\alpha(z)$ is related to the intensity gain $g$ of the material using

$$
\alpha(z)=\frac{1}{2} \Gamma(z) g
$$

where $\Gamma(z)$ represents the confinement factor of the waveguide. In the following computations, we will use a normalized confinement factor such that in the high gain region, $\Gamma(z)=$ $\Gamma_{+}=1$, and in the low gain region $\Gamma(z)=\Gamma_{-}$. Fig. 8 plots the gain margin $\Delta g_{\mathrm{th}}$ versus $\Gamma_{-} / \Gamma_{+}$for two different values of $\kappa L$ and two different values of the duty cycle $\Lambda_{+} / \Lambda$. As can be seen, use of the model in (34) coupled with the reduction of the duty cycle results in a noticeable increase in the gain margin, particularly for deep gratings, a result which confirms the findings of [14]. It should be emphasized here that the analysis of configurations like that shown in Fig. 7 are difficult if not impossible to perform using simple techniques such as coupled-mode theory.

\section{CONCLUSION}

In this paper, we have generalized the RGFM to allow fullwave analysis of fields internal and external to an inhomogeneous DFB laser cavity. A detailed mathematical development of the approach as well as comments concerning its practical implementation have been provided. The key strength of the

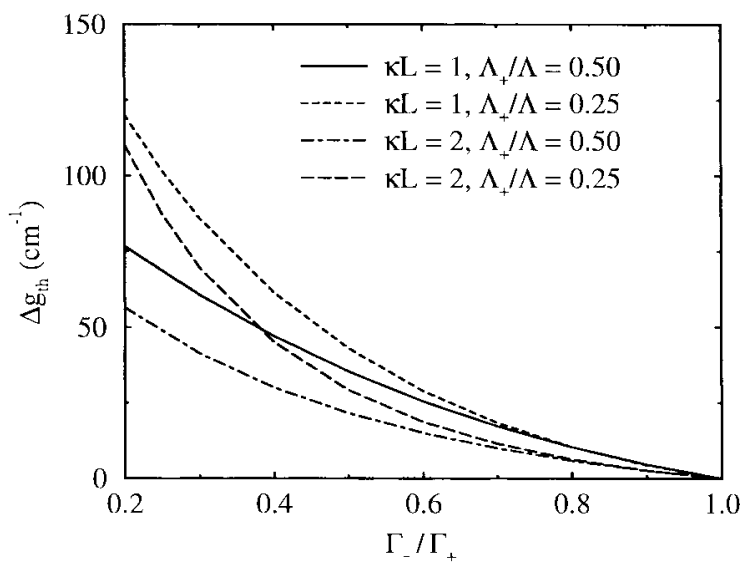

Fig. 8. Single-mode gain margin versus gain grating depth for the complex-coupled DFB structure with different values of gain length $\Lambda_{+}$and index grating depth $\kappa L$.

method is the fact that virtually any device can be modeled using the same algorithm by simply changing the functional form of the material complex permittivity. This advantage is particularly important for devices such as the reduced duty cycle configuration considered, where simplified theories such as coupled-mode analysis are not readily applicable. This flexibility has been demonstrated through application of the method to several representative DFB laser configurations. This analysis revealed the high single-mode gain margin and increased immunity to difficulties associated with SHB from the chirped-grating and MSW geometries. It also illustrated the increase in single-mode gain margin obtained by reducing the duty cycle of the high gain region in complex-coupled DFB devices.

It is anticipated that this generalized RGFM will not only serve as a powerful simulation methodology for sophisticated geometries, but will also serve as a stepping stone for the development of recursive formulations in higher dimensions. Such an extension would allow improved modeling capabilities for many interesting and complex DFB geometries. This work will be the subject of a future publication.

\section{APPENDIX}

Consider finding a homogeneous solution to (2) in the domain $\Omega_{1}$ which satisfies a homogeneous Neumann boundary condition at its left end $z=L_{0}$ but not at its right end $z=L_{1}=z_{B}$. We must first recognize that each of the two forms given in (24) are homogeneous solutions to the wave equation by themselves and must be combined in the manner implied in (24) in order to provide a particular solution to (2). Additionally, the top expression in (24) only satisfies the homogeneous Neumann boundary condition at $z=L_{1}=z_{B}$, while the bottom expression only satisfies it at $z=z_{0}$. As a result, if we choose $G_{1}\left(z, z_{B}\right)$, which means that $z<$ $z^{\prime}=z_{B}$, then clearly we have a homogeneous solution to the differential equation which only satisfies the boundary condition at the left end. In the domain $\Omega_{2}, G_{2}\left(z, z_{B}\right)$ provides a homogeneous solution which satisfies the Neumann condition only at the right end as desired. 


\section{REFERENCES}

[1] H. Kogelnik and C. V. Shank, "Coupled-wave theory of distributed feedback lasers," J. Appl. Phys., vol. 43, pp. 2327-2335, May 1972.

[2] K. B. Kahen, "Analysis of distributed-feedback lasers using a recursive Green's functional approach," IEEE J. Quantum Electron., vol. 29, pp. 368-373, Feb. 1993.

[3] $\_$, "Analysis of distributed-feedback lasers: A recursive Green's function approach," Appl. Phys. Lett., vol. 61, pp. 2012-2014, Oct. 1992.

[4] - "Green's functional approach to resonant cavity analysis," IEEE J. Quantum Electron., vol. 28, pp. 1232-1235, May 1992.

[5] G. Bjork and O. Nilsson, "A new exact and efficient numerical matrix theory of complicated laser structures: Properties of asymmetric phaseshifted DFB lasers," J. Lightwave Technol., vol. LT-5, pp. 140-146, Jan. 1987.

[6] T. Makino, "Threshold condition of DFB semiconductor lasers by the local-normal-mode transfer-matrix method: Correspondence to the coupled-wave method," J. Lightwave Technol., vol. 12, pp. 2092-2099, 1994.

[7] M. A. Jensen and J. D. Freeze, "A recursive Green's function method for efficient solution of the volume integral equation," in 1996 USNC/URSI Radio Science Meeting Dig., Baltimore, MD, July 21-26, 1996, p. 28.

[8] P. Zhou and G. S. Lee, "Mode selection and spatial hole burning suppression of a chirped grating distributed feedback laser," Appl. Phys. Lett., vol. 56, pp. 1400-1402, Apr. 1990.

[9] _ "Phase-shifted distributed feedback laser with linearly chirped grating for narrow linewidth and high-power operation," Appl. Phys. Lett., vol. 58, pp. 331-333, Jan. 1991.

[10] P. Correc, "Stability of phase-shifted DFB lasers against hole burning," IEEE J. Quantum Electron., vol. 30, pp. 2467-2476, Nov. 1994.

[11] Y. Nakano and K. Tada, "Analysis, design, and fabrication of GaAlAs/GaAs DFB laser with modulated stripe width structure for complete single longitudinal mode operation," IEEE J. Quantum Electron., vol. 24, pp. 2017-2033, 1988.

[12] H. Soda, Y. Kotaki, H. Sudo, H. Ishikawa, S. Yamakoshi, and H. Imai, "Stability in single longitudinal mode operation in GaInAsP/InP phaseadjusted DFB lasers," IEEE J. Quantum Electron., vol. QE-23, pp. 804-814, 1987.

[13] J. Whiteaway, G. H. B. Thompson, A. Collar, and C. Armistead, "The design and assessment of $\lambda / 4$ phase-shifted DFB laser structures," IEEE J. Quantum Electron., vol. 25, pp. 1261-1279, 1989.

[14] J. Chen, A. Champagne, R. Maciejko, and T. Makino, "Improvement of single-mode gain margin in gain-coupled DFB lasers," IEEE J. Quantum Electron., vol. 33, pp. 33-40, Jan. 1997.

[15] J. Mathews and R. L. Walker, Mathematical Methods of Physics. New York: Benjamin, 1970.
[16] C. A. Balanis, Advanced Engineering Electromagnetics. New York: Wiley, 1989, ch. 14.

Jim D. Freeze (S'95-M'96) received the B.S. and M.S. degrees in electrical and computer engineering from Brigham Young University, Provo, UT, in 1991. He is currently pursuing the Ph.D. degree at the same institution.

His research interests include mathematical and numerical modeling of electromagnetic wave propagation

Michael A. Jensen (S'93-M'95) received the B.S. (summa cum laude) and M.S. degrees in electrical engineering from Brigham Young University (BYU), Provo, UT, in 1990 and 1991, respectively, and the Ph.D. in electrical engineering from the University of California, Los Angeles (UCLA), in 1994

From 1989 to 1991, he was a Graduate Research Assistant in the Lasers and Optics Laboratory at BYU. In 1990, he received a National Science Foundation Graduate Fellowship. From 1991 to 1994, he was a Graduate Student Researcher in the Antenna Laboratory at UCLA. Since 1994, he has been an Assistant Professor in the Electrical and Computer Engineering Department and Brigham Young University. His main research interests include radiation and propagation for personal communications, numerical electromagnetics, optical fiber communication, and implementation of finitedifference schemes on massively parallel computer architectures.

Dr. Jensen is a member of Eta Kappa $\mathrm{Nu}$ and Tau Beta Pi.

Richard H. Selfridge (M'87) received the B.S. degree in physics from California State University, Sacramento, in 1978, and M.S. and Ph.D. degrees in electrical engineering from the University of California, Davis, in 1980 and 1984 , respectively.

He has been a Professor of Electrical Engineering at Brigham Young University since July of 1987. From 1983 to 1987, he taught in the Department of Electrical and Computer Engineering at California State University, Sacramento. His research interests have included modeling of conduction processes in semiconductors and mode locking of argon and dye lasers. Recently, his research is directed toward the invention and fabrication of novel D-fiber devices. In addition, he is experimenting with approaches for teaching and using differential forms for electrical engineering.

Dr. Selfridge is a member of Sigma Xi and SPIE. 